\title{
Patient Health Monitoring System Using Blue Eye Technology and loT
}

\author{
Abhijeet Ramesh Thakare ${ }^{1^{*}}$ and Nilesh Korde ${ }^{2}$ \\ ${ }^{1,2}$ Shri Ramdeobaba College of Engineering and Management, Nagpur, India
}

\section{ABSTRACT}

Technology has now advanced enough for us to sit in front of our personal computer that can feel and monitor human feelings known as "BLUE EYE TECHNOLOGY." The devices which are used in this technology can detect the emotional level of the human body, such as facial and voice recognition, etc. The equipment used in Blue Eye Technologies is capable of recognizing human movements, checks our personality, senses our presents, and begins to communicate with us. In today's world, HealthCare has become one of the essential things in our life. But, the traditional healthcare system faces various problems, especially in the COVID-19 pandemic. Different challenges are faced by the HealthCare domain like scarcity of HealthCare professionals in hospitals; very expensive treatment, lack of healthcare knowledge, and Manual monitoring of patient's health parameters. In this paper, a remote patient monitoring system is proposed which can monitor patients remotely using Blue Eye Technology and IoT. Doctors can monitor effectively patient's moods and emotions (such as sad, joyful, and surprised) by utilizing the Emotion Sensory System of Blue Eye Technology as well as extract Physiological information of the patients such as Blood Pressure, Heart Rate, Temperature, ECG, etc. This information is effectively used by the Doctors for diagnosis and proper treatment of patients.

\section{KEY WORDS: BLUE EYES TECHNOLOGY, CLOUD, IOT, HEALTH CARE MANAGEMENT, SENSORS.}

\section{INTRODUCTION}

Think about a world where people are communicating with machines. With this capability, machines can interact with human beings using technology like voice and face identification, etc. At the tip of mouse feelings of human beings can also be recognized. The human sense is mainly based upon the ability of audiovisuals and sensors to sense view and incorporate. The BLUE EYES technology is aimed at developing digital devices with visual and sensory capacities close to those of humans. This utilizes a non-obtrusive sensing system, using most conventional

\section{ARTICLE INFORMATION}

*Corresponding Author: thakarear@rknec.edu

Received 17th Oct 2020 Accepted after revision 30th Dec 2020

Print ISSN: 0974-6455 Online ISSN: 2321-4007 CODEN: BBRCBA

Thomson Reuters ISI Web of Science Clarivate Analytics USA and Crossref Indexed Journal

\section{Clarivate
Analytics}

NAAS Journal Score 2020 (4.31)

A Society of Science and Nature Publication,

Bhopal India 2020. All rights reserved.

Online Contents Available at: http//www.bbrc.in/

Doi: http://dx.doi.org/10.21786/bbrc/13.14/3 video cameras and microphones to recognize the actions of the operator by utilizing sensory capabilities provided. By realizing the emotional or physical situation, the machine will give an appropriate reaction and also identify what is expected from the user (Farzana et.al, 2019). As shown in Fig. 1, an IoT network consists of sensors/devices that "connect" through some kind of connection to the cloud.

If the data enters the server, the app stores it and can then agree to execute an operation, such as delivering a message or changing the sensors/devices automatically without the user needing to. In almost every country, manual methods are utilized for the measurement of various patient health parameters like ECG, Temperature, blood pressure, and Heart Rate (Kumawat et. al, 2018). Also, various hospitals utilize extra HealthCare Software for HealthCare Monitoring of the patients. But, this kind of software provides low reliability and accuracy (Rahman et. al, 2019). Nowadays, Especially in COVID-19 pandemic, a huge number of peoples require healthcare support. But, healthcare systems are going through various problems

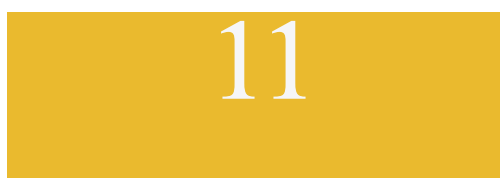


like less number of healthcare workers and professionals in hospitals, very high cost for treatment, and lack of healthcare knowledge (Oyebola\&tToluwani, 2018- Gomes et. al, 2015). So, the main motivation for designing our system is automatic monitoring of the patient's health related parameters from a remote location.

Related Works: In (Dhinakaran et. al, 2020), proposed an Automated Patient Monitoring using Blue Eye Technology (BET) and Cloud Computing. This system is utilized to measure various health parameters of the patients. A "KHealth"- which is a digital healthcare system used for diseased monitoring. This system (Sharma et. al, 2018) is analyzed for Practical Privacy-Preserving Analytics. A potential tradeoff in privacy is also discussed in this work. IOT also plays a major role in health monitoring. By combining IoT with Blue Eye Technology, highly reliable data transmission is achieved.

Various IoT devices generate big amounts of data. This data is further used for analytics purposes by applying different Artificial Intelligence Techniques (Bravo et. al, 2011). Cloud Computing also plays a crucial role in health monitoring. Various services are provided by Cloud Computing. For example, Analytics, Database Storage, Servers, and different kinds of Intelligence over the Internet (Yi \& Saniie, 2016). The system proposed in (Wu et. al, 2019) utilizes a wearable body area network (WBAN) for collecting user data and a low-power widearea network (LPWAN) for collecting the WBAN with the Internet. In this work, a standalone local server (gateway) is used, which can analyze the raw sensor signals and display the environmental and physiological data. It also triggers an alert message if any emergency circumstance is detected for the patient.

Figure 1: Internet of Things (IoT)

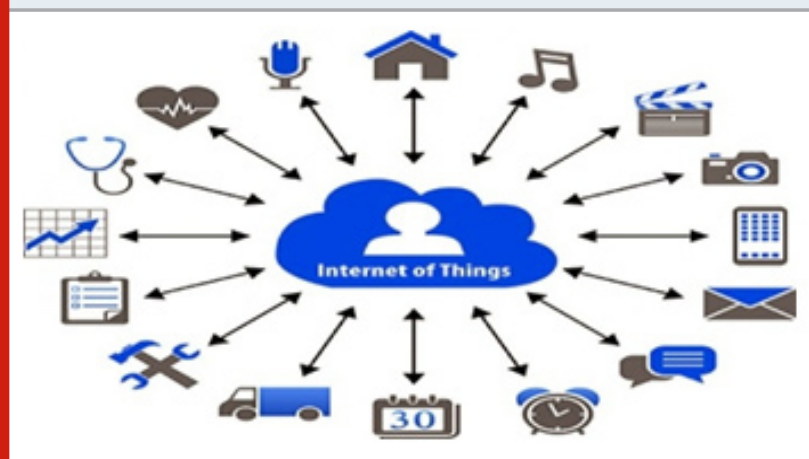

Proposed System: Our Proposed system (Figure 2) comprises three main components which are as follows:

1. Data Acquisition Unit (DAU) 2. Central System Unit (CSU) 3. Software

Data Processing Unit: Data Processing Unit consists of two units:-

Data Acquisition Unit (DAU): Data Acquisition unit is one of the chunks of the Blue Eyes System. The key function is to collect the sensor's physiological data and transfer it to the central device for processing. The Data Acquisition Kit contains numerous Atmel 89C52Microcontroller hardware modules, Bluetooth core part (which is based on ROK101008), HD44780 - tiny LCD 24C16 I2C EEPROM, Jazz Multisensory interface, Indicators comprises of beeper and LED and 6 AA batteries and voltage monitoring capabilities.

Central System Unit (CSU): It is an equipment for Central Network System is the wireless link per second. The package includes a Bluetooth module and a speech data transfer PCM codec. The module uses a parallel, serial, and USB cable to connect to a PC. By utilizing standard mini-jack sockets audio data is accessed.

The Software: The primary role of Blue Eye System Technology is to look after the medical state of the operators which is operating. Actual time tendering and analysis of incoming as well as physiological data to display instance response on the state of Operator, warning firing is performed by the program. The system center with many usable modules is a Blue-Eyes program that supports the transition flow between other device modules (e.g raw data fetching from the Connection Manager to data analyzers, forwarding interpreted data from data analyzers to files, GUI controls, and other data analyzers). Visualization software provides managers with user experience. It is possible to set the Visualization application in an off-line domain. In this case, all the data is fetched from the database. The instructor reconstructs the details selected from the course. Adding exceptional perceptive capabilities to computers will require computers to function as intimate partners with human beings.

This software enables an overview of the chosen video source and associated sound stream monitoring of the operating operators to function as intimate partners with human beings.This software enables an overview of the chosen video source and associated sound stream monitoring of the operating operator's Physiological state. On the incoming warning messages, the Supervisor is signaled immediately each time. An attempt of attaching additional functionality to computers are performed by researchers which permit computers to be communicated as a human being, recognize human objects and also listen to guess, speak, and even listen to human sentiments. It aims to build virtual machines with visual and sensory capacities close to those of humans. The task of the Operator is to watch all the physiological conditions, warnings, video, and audio data captured. Physiological data are displayed using a series of custom-built GUI functions.

\section{Emotional Sensors:}

Emotional Sensors Used for Hand:

Emotion Mouse: The working of Emotion Mouse are movements of the mouse, recognizing finger force when the user presses the mouse button, realizing mouse button click frequency, Extracting various Physiological 
information parameters like ), Electromyography activity, Skin temperature, Skin electricity, and Heart rate (ECC/ EKG).

Sentic Mouse: It is a revised version of a computer mouse that comprises a directional pressure sensor and it is also utilized to help in emotional valence recognition (liking/ attraction vs. disliking/avoidance).

Figure 2: Overview of the System

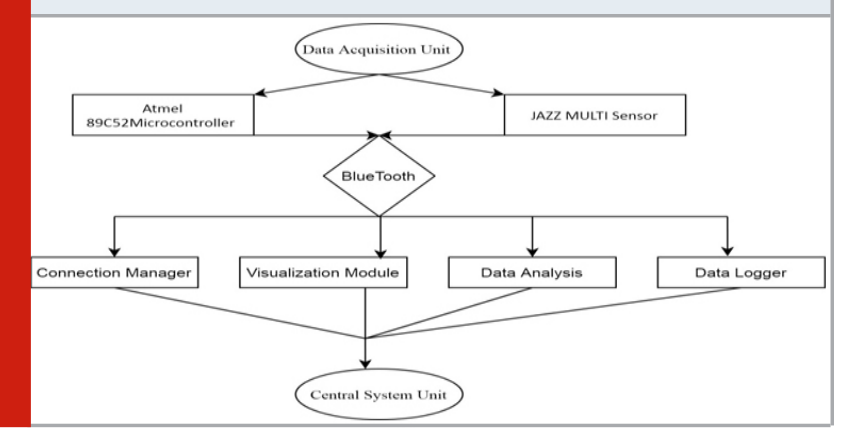

Emotional Sensors Used for Eyes:

Expression Glasses: An interactive tool that helps the user to imagine the wearer's discomfort and excitement rates. Numerous ongoing advances in applied innovations incorporate the endeavor to comprehend the client's advantages just by watching the client PC interface to make sense of what the person is enthusiastic at a particular time. For example, by extracting the person's mood by identifying the type of website e opens/surf, the machine might scan similar websites and predict the user's results.

Magic Pointing; This research explores a new approach of using eye contact for feedback from computers. Gaze tracking has long been used as an alternate or theoretically superior pointing tool for machine input. Two different MAGIC pointing methods, one conservative and one radical have been developed, evaluated, and applied using an eye tracker.

\section{Emotional Sensors Used for Voice:}

Artificial Intelligence speech Recognition: Consideration of the context in which the speech recognition system will operate is essential. Artificial Intelligence (AI) has two concepts. First of all, it involves researching the human being's cognitive processes. Then the second is that it involves using devices (like computers, robots) to represent certain systems. AI has also taken the machines smart and made them more useful and less costly than human intelligence. Natural language processing (NLP) is one of the techniques used for artificial intelligence to interact with a computer in a human language such as English. The NLP program takes the data, reads it, and launches operation. Input words are checked and balanced against known words that are processed internally. Acknowledgment of a keyword initiates any practice. That is the way, a person can interact with his or her own machine Language, no special commands or programming languages are needed and, thus, there is no need to write the script in a different language.

Patient Monitoring Scenario: Figure 3 depicts a scenario in which Blue Eye Technology and IoT are utilized for patient monitoring. As shown in Figure 3, A Patient Suffers from Some Diseases like Heart Attack, So We place a Sensors on her Body and a Mobile device can serve as a gateway between the Wireless Body Area Network and the IoT cloud. It is implemented control of power networks using embedded Web servers. To monitor body temperature and heartbeat, wearable sensor nodes can be placed on the outside of the body in various positions. Falls are tracked in the event of an emergency by using an accelerometer on the node. Blue Eyes utilizes software, cameras, and sensors for voice recognition to recognize and respond to emotional levels and the ability to detect subtle variations in human moods.

Biometric sensors are utilized for calculating and investigating various physiological expressions related to feelings. For Example, if the patient is in critical condition, then we will give the health tracking website a warning letter. Using GPS, we can also track the location of the patient. One of the uses of wireless patient health monitoring is that if any individual has a heart attack, the person who is closest to the patient after some time is identified. To keep us from tracking the patient's body parameters continuously, real-time data collection of patients along with assistance by medical professionals is possible by fully eliminating the manual data collection and tracking the enormous amount of patient information. One of IoT's uses is to make healthcare devices more effective by constantly tracking patient health, through which the sensor obtains patient data and we can minimize human error by supporting this method.

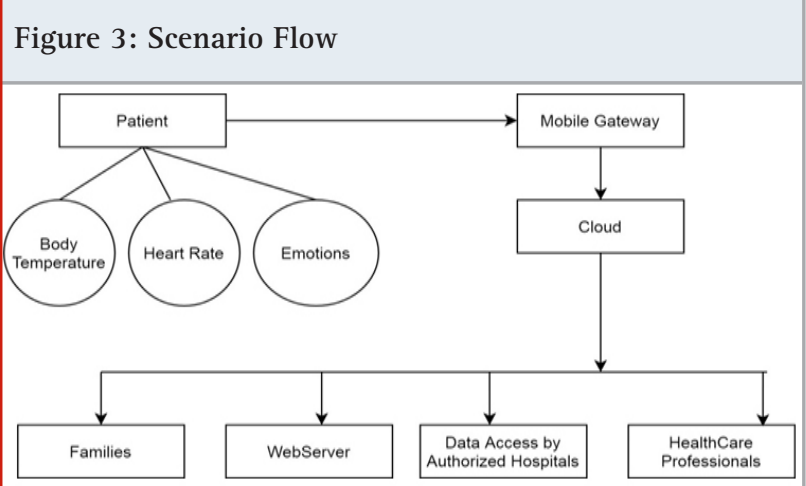

Implementation: The development of the framework in the clinic includes developing of disengaged wards for every patient who gets conceded and looks for a concentrated oversight. The patient is encircled by a day in and day out checking component which comprises of a high-end camera. This camera consists of a CMOS Sensor [Rahman et. al, 2019] having characteristics of elevated quantum potency. This characteristic will be useful for the remarkable sensing of a patient. To sense the feeling intensity of the patient, a sentic mouse is kept in a visible network with the hand of the victim patient. 
The sentic mouse has a collection of sensing elements that includes, an intrinsical pressure sensor accustomed notice the blood pressure, a Galvanic Skin Response sensor - measures the duct gland activities, a temperature sensor - permits body temperature measuring associate degreed a photograph sensor - confirms the absence or presence of an object. AD 8232 graph sensing element is placed on the patient's body surface nearer to the heart for regular measurement of rhythm. Also, a pulse rate sensor is strapped around the patient's chest to record the patient's heart rate in real-time. An electroacoustic transducer is employed to enable the patient's speech recognition Table 1 describes the reading of two categories of patients. One is Male and another is Female. These readings are taken for every patient after a time interval of 3 hours. Various Health parameters are sensed remotely using our implemented system.

Table 1. Patient Parameter Investigation

\begin{tabular}{|c|c|c|c|c|c|c|c|c|c|}
\hline ID No. & $\begin{array}{c}\text { Age } \\
\text { (In Years) }\end{array}$ & $\begin{array}{l}\text { Gender- } \\
\text { Specific }\end{array}$ & $\begin{array}{c}\text { Date } \\
\text { (DD-MM-YYY) }\end{array}$ & $\begin{array}{c}\text { Time } \\
(\mathrm{AM} / \mathrm{PM})\end{array}$ & $\begin{array}{c}\text { Temperature } \\
(\% \mathrm{~F})\end{array}$ & $\begin{array}{c}\text { Blood } \\
\text { Pressure } \\
(\mathrm{mm} \mathrm{Hg})\end{array}$ & ECG & $\begin{array}{l}\text { Heart } \\
\text { Rate }\end{array}$ & Emotion \\
\hline \multirow[t]{3}{*}{1} & 25 & $\mathrm{~F}$ & $24 / 05 / 2020$ & $11.00 \mathrm{AM}$ & $97.2^{\circ} \mathrm{F}$ & 118/79 mm Hg & Regular & $75 \mathrm{bpm}$ & Sadness \\
\hline & & & & $2.00 \mathrm{PM}$ & $97.2^{\circ} \mathrm{F}$ & $121 / 85 \mathrm{~mm} \mathrm{Hg}$ & Regular & $83 \mathrm{bpm}$ & Sadness \\
\hline & & & & $5.00 \mathrm{PM}$ & $98.2^{\circ} \mathrm{F}$ & $125 / 88 \mathrm{~mm} \mathrm{Hg}$ & Regular & $88 \mathrm{bpm}$ & Happiness \\
\hline \multirow[t]{3}{*}{2} & 60 & M & $24 / 05 / 2020$ & $11.00 \mathrm{AM}$ & $99.2^{\circ} \mathrm{F}$ & 133/80 mm Hg & Regular & $90 \mathrm{bpm}$ & Fear \\
\hline & & & & $2.00 \mathrm{PM}$ & $99.6^{\circ} \mathrm{F}$ & $137 / 86 \mathrm{~mm} \mathrm{Hg}$ & Regular & 93 bpm & Disgust \\
\hline & & & & $5.00 \mathrm{PM}$ & $99.9^{\circ} \mathrm{F}$ & $140 / 90 \mathrm{~mm} \mathrm{Hg}$ & Irregular & $101 \mathrm{bpm}$ & Anger \\
\hline
\end{tabular}

\section{CONCLUSION}

The BLUE EYES system makes the machine so smart and knowledgeable it behaves like a human being. By offering more comfortable and user-friendly services in electronic apps, it makes human life easier. we've shown the approach so far, the next step is hardware enhancement. Now, that we have proved the process, instead of using bulky devices to gather user information, the use of smaller and less invasive systems would be preferred. the day isn't far from this development working its way into your home keep, making you lazier. It can even touch a mobile device that holds your hand. Blue Eyes Technology along with IoT can be effectively used to remotely monitor the health condition of patients. Health data generated in this system is extracted and analyzed by Health care professionals, Doctors, hospitals for further diagnosis and treatment of patients.

\section{ACKNOWLEDGEMENTS}

The authors would like to thank the Department of Computer Application and the Department of Computer Science and Engineering at Shri Ramdeobaba College of Engineering and Management Nagpur.

\section{REFERENCES}

Bravo, I., Baliñas, J., Gardel, A., Lázaro, J.L., Espinosa, F. and García, J., (2011) Efficient smart CMOS camera based on FPGAs oriented to embedded image processing. Sensors, 11(3), pp.2282-2303.

Dhinakaran, K., Nivetha, M., Duraimurugan, N. and Wise, D.J.W., (2020, July) Cloud based Smart Healthcare Management System Using Blue Eyes Technology. In 2020 International Conference on Electronics and Sustainable Communication Systems (ICESC) (pp. 409-
414). IEEE.

Farzana, A., Arshadh, H., Ganesan, J. and Muthukumaran, M., (2019) High Performance VLSI Architecture for Advanced QPSK Modems. Asian Journal of Applied Science and Technology (AJAST) Volume, 3, pp.4549.

Gomes, Y.F., Santos, D.F., Almeida, H.O. and Perkusich, A., (2015, January) Integrating MQTT and ISO/IEEE 11073 for health information sharing in the Internet of Things. In 2015 IEEE International Conference on Consumer Electronics (ICCE) (pp. 200-201). IEEE.

Kumawat, M., Mathur, G. and Saju, N.S., (2018). Research Paper on "Blue Eye Technology".

Oyebola, B. and Toluwani, 0., (2018) Blue Eyes Technology In Modern Engineering: An Artificial Intelligence. International Journal of Higher Education, pp.45-65.

Rahman, A., Rahman, T., Ghani, N.H., Hossain, S. and Uddin, J., (2019, January) IoT Based patient monitoring system using ECG sensor. In 2019 International Conference on Robotics, Electrical and Signal Processing Techniques (ICREST) (pp. 378-382). IEEE.

Sharma, S., Chen, K. and Sheth, A., (2018) Toward practical privacy-preserving analytics for IoT and cloudbased healthcare systems. IEEE Internet Computing, 22(2), pp.42-51.

Wu, F., Wu, T. and Yuce, M.R., (2019) An internet-ofthings (IoT) network system for connected safety and health monitoring applications. Sensors, 19(1), p.21.

Yi, W. J and Saniie, J., 2016. Patient centered realtime mobile health monitoring system. E-Health Telecommunication Systems and Networks, 5(04), p.75. 